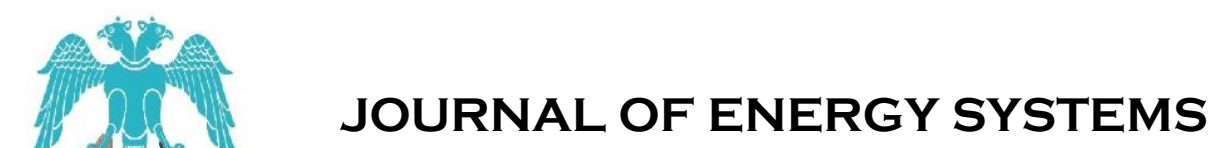

Volume 2, Issue 1

DOI: $10.30521 /$ jes.405774

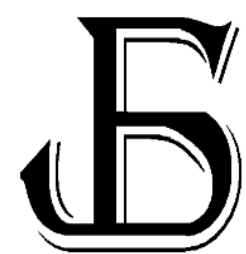

2602-2052

Research Article

\title{
A Low cost modelling of the variable frequency drive optimum in industrial applications
}

\author{
Ahmed Ali \\ Gaziantep University, Electrical and Electronics Engineering Department, 27310, Gaziantep, Turkey, \\ engineer28ahmed@gmail.com, orcid.org/0000-0002-0352-6644 \\ Ergun Erçelebi \\ Gaziantep University, Electrical and Electronics Engineering Department, 27310, Gaziantep, Turkey, \\ ercelebi@gantep.edu.tr, orcid.org/0000-0002-4257-3531
}

Arrived: 14.03.2018 Accepted: 25.03.2018 Published: 31.03.2018

\begin{abstract}
A single or three phase asynchronous motors, or known a squirrel cage motors (SCMs) are represent one of generally using in many industrial applications. They are consuming further than half-percent of the total generated electrical energy. This motor is working at its full speed when it is attached to the main AC- supply. Therefore, speed control of asynchronous motor is necessary to industrial applications that require changeable flow control of fluid (air, water and chemical liquid streaming). There are many methods to control the speed of motors, such as changing the stator number of poles, controlling supply voltage, addition series reactor or resistances. The modern method is Volt per Hertz, or scalar control this method applied by changing voltage and frequency of three-phase supply, or by making a V/f equal to a constant value in this method a large amount of energy can be saved.

In this study, analysis and practical implemented to variable voltage Variable Frequency Drive or called a VFD. The model enables an exception of a large amount of energy, when connected to control speed of Induction motor. A new simplified method for implemented of an AC Drive system with a moderate price for components was proposed. This method totally based on assembling overall components of AC-Drive. The advantage of this design is simplicity, robustness and ease of tuning as well as manufacturing due to these advantages. This design is very suitable for real time applications as motor speed controller. The experimental results obtained show that design helps to preserve a large amount of energy to the power grid, by a limited starting current of the motor as well, eliminating a voltage flicker at starting and reducing dissipating power during running in long period. Results verified experimentally
\end{abstract}

Keywords: Variable Frequency Drive, AC-Drive, Induction motor, Volt per Hertz,

Cite this paper as: $\quad$ industrial applications. Journal of Energy Systems 2018; 2(1): 28-42, DOI: 10.30521/jes.405774

(C) 2018 Published by peer-reviewed open access scientific journal, JES at DergiPark (www.dergipark.gov.tr/jes) 


\section{INTRODUCTION}

The DC-Motors and surreal cage induction motors (SCIM) are one of main types of motors that used in many industrial applications. The induction motors, mainly the asynchronous motor or known as squirrel cage type is the most frequently used in industry owing to their compensation more than the DC-Motors. The biggest benefit is that induction motor (IM) does not need an electrical bond between motionless and rotating portion of the motor. Hence, they perform not require any automatic commutator and brushes leading actuality that they are maintenance free. Induction motors are more economic over DCMotor and it has robustness, strong makeup, elevated efficiency (approaches to 85\% or more) in addition the IM be able to work in hotheaded environments since has no brushes, consequently no sparks are formed [1][2] The extensively developments in power electronics module and power semiconductor strategy, which guide to replace the main changeable speed applications use DC-Machine by ACMachine, AC- Drives replaced DC-Drives and at present time they are prevalent use manufacturing and home applications. Though, changeable speed drive system (VSD) to AC-Machines are an imperative part in each industrial processes they are a perfectionist and help to receiving an optimized method by dipping an energy and operating expenses. There are three typical (VSD) systems; mechanical drives, electrical drives, and hydraulic drives. In update paper discusses electrical drive. The electrical AC Drive is an electronic device that provides regulation into the speed of AC-Machines [3] (see Figure 1) [1].

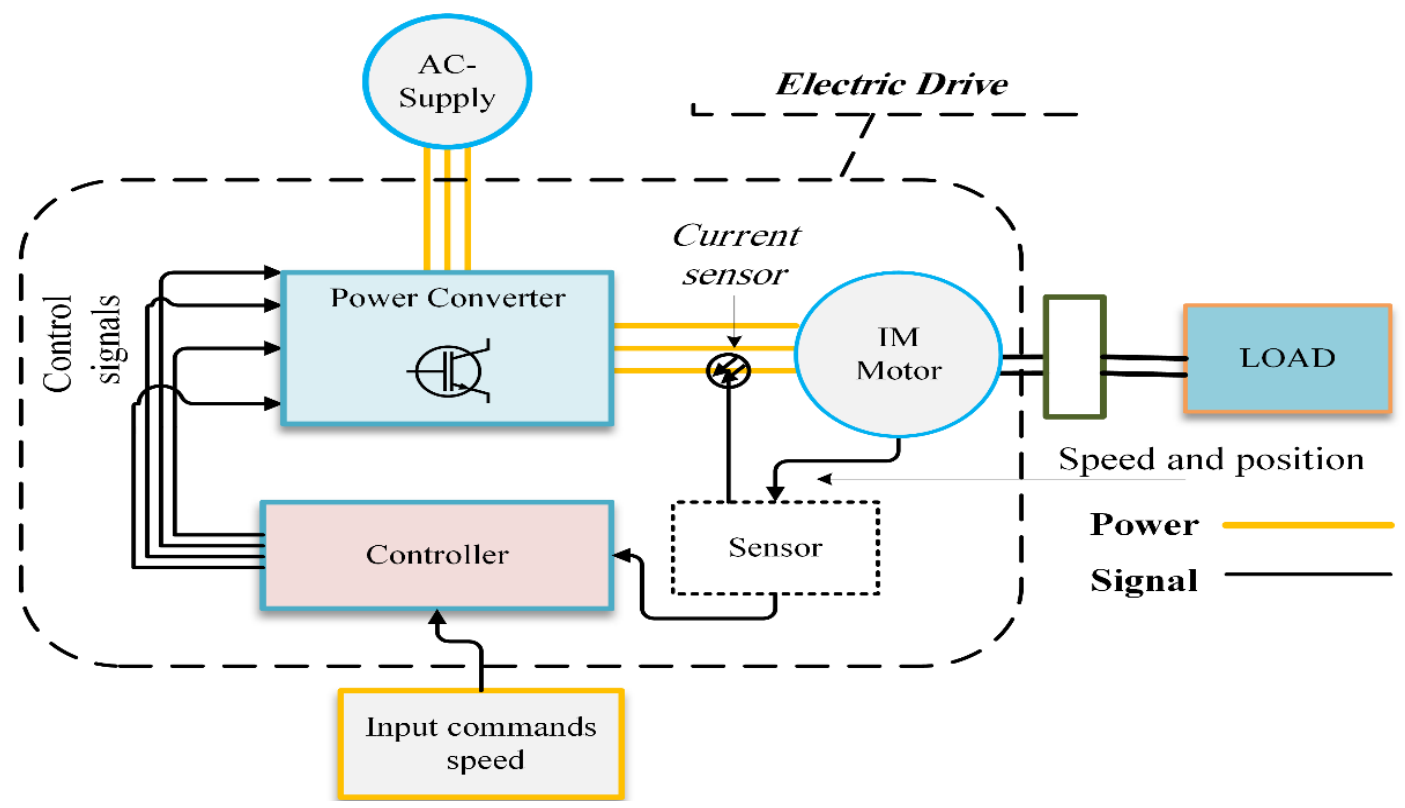

Figure 1. Electric variable speed drive (VSD) system scheme diagram.

There are many papers published in the field of energy saving by using VFD a few papers focused to investigate on the power management and developing a new strategy to regulate active and reactive power flow to the motors. In [4], a new flexible approach to manage active and reactive power, such that the optimal torque-speed profile of the turbine be able to be followed and total reactive power is prearranged, while the machine copper losses have been minimized. Simultaneously, eliminating Harmonic that's injected into the power system. In this advance, the systems are able to utilizing as in cooperation a high-efficient power generator and a flexible reactive power compensator. According to the paper [5] developed novel, fast, and accurate technique for measurement iron losses in enduring magnet synchronous machines with introduced dedicated laboratory classification for acting automatic measurement. The attitude of the measurement method, the main building blocks of the laboratory system and the procedures of tests are studied. Additionally, a method for the separation of the iron loss 
into hysteresis and eddy current components, the development of the change in the magnetic flux density due to changeable temperature of the rotor permanent magnets and the calculation of the immediate permanent magnet temperature are accessible briefly. In [6] a comparison stuck among ON-OFF and VFD scheme to manage ventilation fans of glass house and chicken houses has been, accompanied. The paper focused to define achieve of each system on the energy expenditure and consequential microclimate inside the houses. The real time test was conceded out in a trade -size glass house in which pepper was grown-up and in trade fowl houses. Rising the fan speed with the variable occurrence drive system resulted in reductions in the airflow speed throughout the houses and energy use. This paper indicated that VFD control could decrease electricity expenditure compared with ON-OFF process. Eventually obtained in the glass house additional, that the VFD system has an inordinate possible than the ON-OFF to modest the range of amplitude variations in the air hotness and dampness ratio within the greenhouse. Nevertheless, noticeably lower with VFD than ON-OFF. Reductions in the variety of amplitude variations of the air hotness and humidity were also experimental in the chicken houses. Practically motors are designed to run at a constant speed and produced a constant output. Hence, new technology has need of diverse speeds in various applications where electric motors are used. In [7] the Authors are supportive to provide an extensive review on applications of VSD in electrical motors energy savings.

The objective is to recognize the origin of energy saving opportunities and incorporated costs of installing variable speed drives to the existing applications of electrical motors. Afterward, economic scrutiny, reimbursement period and the effect of current and voltage harmonics generated by variable Speed Drives are obtainable. In [8] obtainable a classification of the current market of the VSDs including the average prices, installation expenses, and the whole sales in to European Union countries, based on the power rating feature, also in this paper the input barriers to inclusive application of VSDs are documented. In exacting, the power quality and dependability problems associated with the use of VSDs are discussed the promising technical solutions [9] provide recommendations on the accurate selection of a VFD in order to employed with a centrifugal pump in to Petroleum Facilities, to provide flow, pressure or temperature control. That paper has covered the effect on the pump- system from point of view commercial considerations of VFD control valve; correct planning and installation considerations to VFD and regulation a control loop when VFD handle as the final control element. As well describe the further advantages, which is a VFD can bring involving elimination of emissions the improved analytical information around the operating motor which can be brought into the control system and the limited wear on bearings and seals in a pump by utilizing a VFD. Even more, presented some application examples on VFD where used as a terminal control element in the petroleum industry in case of a reversed stream at same direction of stream sectors of. In [10] investigates about the measurement power and efficiency to together motor and VFD system, initially presents a general survey to international standards regarding IM and VFD systems, subsequently, confers some of the assignments about the measuring of active power from the PWM- inverter source. Two induction motors were test through two different sources sinusoidal supply VFD supply. Variances in power measurements due to different power analyzer situations are elucidate. Eventually, the results indicate to efficiency tests for the five HP motor have been compared. [11] The paper proposes an essential simplified model of the VFD, which provides a sufficient modelling for the study of electromechanical transients in large-scale power systems. The model has implemented through an open source computer software Power System Toolbox (PST). The protection and control strategies that are allow to given a good performing to the model with severe disturbances has complemented. In addition, the aim of this paper is enhancement of the model parameters as well as at the development of new approaches for VFD protection and control. [12], presented an extensive summary of the component design and system dimension simultaneously with control techniques. Explain and compare the valuation of the flow rate of each pump when the VFD attached to supplying to a parallel pumping system by developed new models. Approximately 30-50\% of energy waste can save with active element and designing the pumping system. In case of the parallel pumping system, variable speed drive makes an available greatest chance to protection energy be save with respect to element selection and size of the pumping system. A comparison of energy-efficient increase on the pumping system with the amount of energy savings associated with each efficiency measure. Nearly 15-25\% of energy can be saved with efficient by suitable dimensions of the design piping system for water pumps are more efficient into energy saving 
until thirty-five Terra Watt hour, through regulation expanded product approach. The centrifugal pumping system with variable frequency drives carrying many advantages as well as some drawbacks. The advantages of vector controls in VFD, if compared with the scalar control, it has a dynamic response for both speed and torque with reduced switching losses, but the drawback of vector control it take higher time for computation. In [13] established a flow meter to a virtual pump which can be achieved through building automation systems with respect to motor mechanical power and pump head. Initial, the motor efficiency regressed as a function of mechanical power by combining multiple parameters, subsequently, a pump efficiency function is reassembled with pump mechanical power and head, and eventually, experiments are carried to evolve and verified the implicit pump water flow meter on a chilled water pump with VFD. The experimental results described that the implicit flow gauges well agree with the flow measurement by a physical meter.

\subsection{Significance of the Study}

In this paper Study, discuss and implementation of AC drive. The presenting integrated VFD system used for controlling the speed of the 3 ph synchronous motor, which is considered the most popular AC - Machines. The practical model imitates to conceptions of the power electronic module. The real time SPWM inverter module "HY-DQ-Inverter 2.0" uses to control of the (1-Hp induction motor). The practical model has been developed through adding extra hardware modules, which improve the performance of inverter, by avoiding the risk of damage in the circuit. The practical model completely implemented and excite results are obtained. The advantages of this model are easy tuning and maintenance, moderate cost and achieved to satisfy the requirements of the user. The analyses based on the energy measured between supply and load side. Two tests presented; the first is by direct connected of 3-phase squirrel cage induction motor $(1-\mathrm{Hp})$ to the sinusoidal power supply, to demonstrate energy measurement. The second test is by connecting practical design of AC Drive, finally a simple comparison between tests.

\subsection{Outline}

This paper depicts the operation principle of the Variable Speed Drive system. Further, the basic block diagram of electric drive system is shown. Subsequently an approach for controlling strategy of VFD and the concept of energy saving in applicable of VFD with a large-scale pump. In the rest section a scalar control system has been implemented. This section, involved a block-diagrams for the proposed Ac-drive system and experimental results of the energy saving conception.

\section{VARIABLE SPEED DRIVE}

In this section conception and an essential components of Variable Frequency Drive will be explained briefly. Nowadays VFD is, famous by various names such as an Inverter, frequency converter or ACDrive. In typically, AC- Drive consists of a power converter module, control circuit and electric motor as shows in Figure.1. The converter controlled to AC power flow to electric motors [1]. The VFD produce three-phase lines supplied to electric motor (see in Figure. 2) A VFD can control to torque, speed as well as provides control on speed of motor at same time improved the system efficacy by saved the potential energy. Rectifiers are used to Rectification three or single phase AC to DC [7] There are two commonly used structure for medium power rectifier units: The Diode bridge and the IGBT rectifiers. The diode bridge rectifier or known as six -pulse uncontrolled rectifier. This configuration is most widespread used in power converter to produce a fixed direct voltage. The power circuit of the bridge rectifier contains six power diodes in a three-phase 3-wire system. This means the Rectification voltage is totally depending on the AC supply voltage. The six -pulse uncontrolled rectifiers are nonlinear loads that is lead to drawn non-sinusoidal current from the grid line [14]. Therefore, the 
disadvantage in large scale rectifier system it necessary to connect a harmonic filter to prevent fluctuation in grid. Inverters generate an AC through sequentially switching strategies a DC to AC circuits through the load [7]. Recent years, all inverters are building with IGBT module the configuration of IGBT module carries of the parasitic body diode. Whilst in absence body diode IGBT wanted a freewheeling diode especially placed across it. The PWM technique is most widely used for control of the power electronic switches. PWM control based on height switching (ON and OFF) the power electronic switches. In such a way that pulses with variable width form a variable waveform. [14] The prevalent block diagram to VFD is shown in Fig. 2.

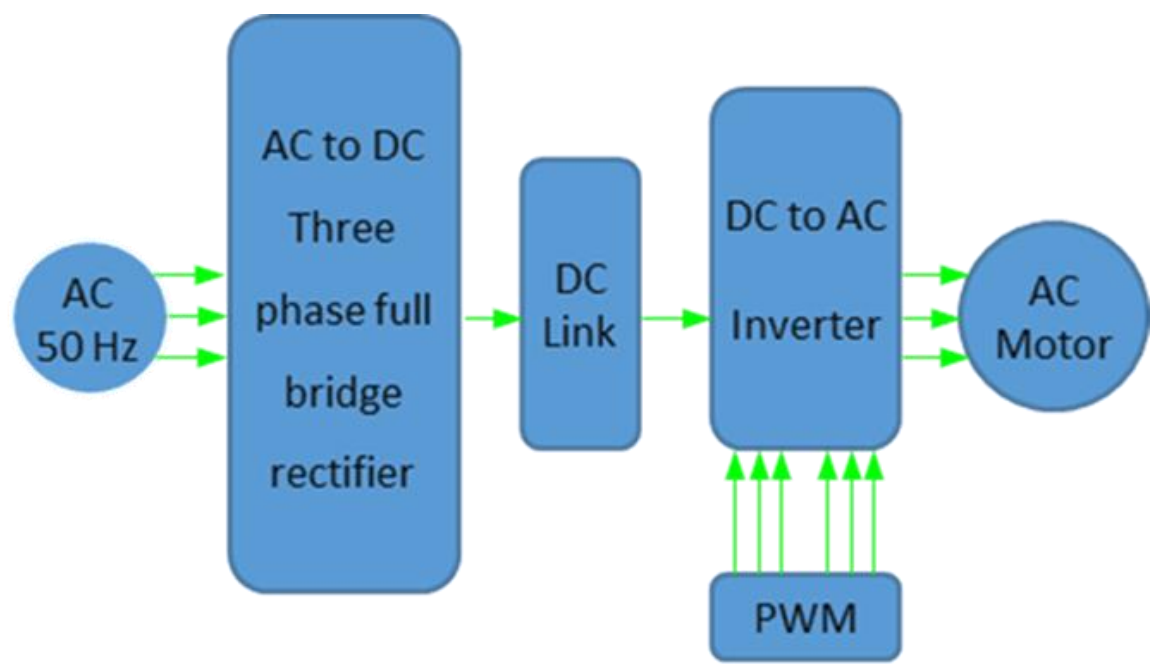

Figure 2. Variable Frequency Drive (VFD) system scheme diagram.

\subsection{Control Type of AC-Drive}

The high performance AC drives, motion control is based on the method of the control which is used and it's necessary to introduce them [14]:

1) Scalar Control strategy

2) Vector Control strategy

3) Direct torque Control strategy

4) Intelligent Control strategy

\subsubsection{Scalar control}

Despite the fact that a controller with a constant voltage of frequency ratio is the simplest controller, it is the most common being in the majority of the industrial applications. It is called as a scalar controller and acts by imposing a constant relation between voltage and frequency or volt/hertz [15].

The structural is very simple and is often used without speed feedback. However, this controller doesn't approach to good accuracy in both speed and torque responses, at most due to the fact that the stator flux and the torque are not directly controlled. However, as long as the parameters are identified, the accuracy in the speed can be within $2 \%$, unless in a very low speed. The basically concept of scaler control strategy illustrated in Figure 3 [1]. The simplest conception to this strategy is based on the ratio (Volt/Hertz) as a constant value by using a one of inverter switching techniques. The switching technique produces a varying pulse width by modifying a duty cycle. The six- PWM signals fed-to gate of each power electronic switches. Which means that produces varying scales in both voltage and 
frequency in output side of inverter. Where, a carrier frequency is defined a speed of turning (ON and OFF) of power electronic switches [16].

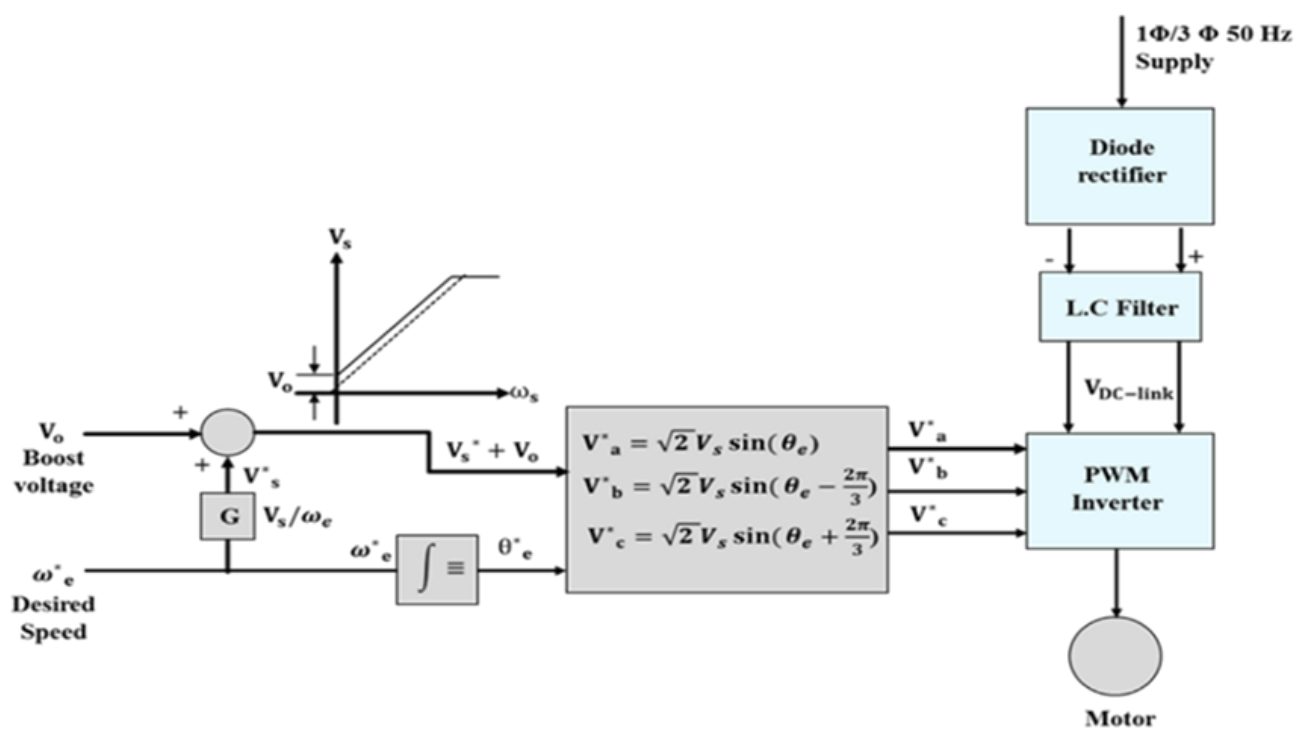

Figure 3. Block diagram of scaler control strategy.

$\theta_{e}$; Electrical Angle of Reference Voltage Vector (rad.). $\mathrm{V}_{\mathrm{a}}{ }^{*}, \mathrm{~V}_{\mathrm{b}}{ }^{*}, \mathrm{~V}_{\mathrm{c}}{ }^{*} ;$ Three Reference signal Sinusoidal Waveforms, $\omega_{e}$; Electrical frequency (rad./sec.), $\omega_{s} ;$ Synchronous Speed (rad./sec.), $V_{s}$; Sinusoidal reference signal voltage $(\mathrm{V}), V_{o}$ Boosting voltage ratio $(\%)$.

\subsubsection{Vector control}

Vector- control strategy, which is also called as Decoupling, Orthogonal, or Field-Oriented Control (FOC), permit the asynchronous motor to be driven with high dynamic performance that is identical to the performance of DC motor [2]. In these strategy of controllers, there are control loops for controlling both torque and the flux. The most common controllers of this type are those that use vector transform such as the Park transform. Its accuracy can reach values such as $0.5 \%$ related the speed and $2 \%$ concerning the torque. The main drawback is huge computational capability required and the obligatory good identification of motor parameters [17].

In this control strategy the 3-phase stator currents are transformed to fictitious two-phase current components. The FOC technique decouples the two components of stator currents: one providing the air-gap flux and the other producing the torque. These current components provide independent control of flux and torque, and the control feature is linearized. These components are transformed back to the stator frame before feeding back to the machine. The two components are d-axis (Direct-Axis) Ids analogues to field current, and q -axis (Quadrature -Axis) Iqs analogues to armature current of a separately excited DC motor. The utility of vector control can be, summarized as follows [18].

1) Full motor torque capability at low speed.

2) Better dynamic behavior.

3) Higher efficiency for each operation.

4) Control of torque and flux.

5) Short term overload capability. 


\section{Direct torque control}

Takahashi introduced a superior scalar control technique, associated as direct torque and flux control (DTFC or DTC). In this method, the motor flux on the stator part and the torque are controlled directly by choosing the suitable state on voltage source inverter [19].

Nowadays (FOC) and (DTC) are considered [20]. The common significant techniques to attain high dynamic performance in AC machines. The main advantages of DTC are: Today (FOC) and (DTC) are considered [20]. The main features of DTC are:

1) In absence of coordinate transform.

2) Absence of voltage modulator block, as well as controllers such as PID for motor flux and torque.

3) Minimum torque response time.

Whereas, some disadvantages as well present such as Requirement of torque, and flux estimator, implying the consequent parameters identification. Inherent torque and stator flux ripple.

\subsubsection{Control intelligent control}

Intelligent control is the ability of a controller to comprehend, reason, and learn about a process. Intelligent control is based on artificial intelligence (AI) which can be realized as computer emulation of the human thinking process. The AI techniques are typically categorized as an expert system (ES), fuzzy logic (FL), artificial neural network (ANN) and genetic algorithm (GA). An expert system concentrated on Boolean algebra in the internal structures and uses hard or precise computation. Whilst fuzzy logic, neural network and genetic algorithm are based on soft or approximate computation. With a control based on artificial intelligence, a system is said to be "intelligent", "autonomous", "adaptive", "self-organizing" or "learning control". The traditional control design is based on a mathematical model of the plant. Usually, the plant model is undiscovered, or ill-defined. Alternatively, the system may be nonlinear and complicated, multivariable with changeable parameter problem. An intelligent control system can recognize the model. if indispensable; give predicted performance also with a large-scale range of parameter variation. Of course, if a model is available it can be used for simulation study while the control can be optimized by iteration. Artificial neural network or neural network (ANN) is the most generic form of AI compared to the expert system and fuzzy logic. Essentially, it is a linkage of the artificial neurons with predominantly nonlinear transfer function at an output. A neural network can be feedforward or feedback (or recurrent) type. Neural network has been used in various control applications in power electronics and drives. Some of these applications are PWM control, on-line diagnostics, direct or inverse nonlinear model emulation of machine, model referencing adaptive control (MRAC), etc. Control of asynchronous motor can be performed in a few milliseconds, by virtue of fast NN methods [20]. Genetic algorithm (GA) presents an efficient parameter identification method for induction machines. GA operates on real-valued parameter sets. Conventional GAs, as stochastic offline optimization algorithms, has been applied to recognize IM parameters .as well as an estimation of mechanical system parameters such as inertia, Coulomb's friction of an inverter-fed IM [21]. Fuzzy logic possesses fair features, so it is insensitive to outside disturbance and small-unknown information. Thus, it deals with problems that have ambiguity or uncertainty and uses membership functions with two value 0 and 1 to compute the problem. A fuzzy controller transforms a set of linguistic rules that based on expert knowledge into an automatic control strategy such as controllers have been found to be excellent for classical controllers, predominately when the information being processed is inaccurate or uncertain. The drawbacks of NN and FL involve the requirement of much training or knowledge base to know the model of a system or a process [22]. Some design path is presented to hybrid drive control system, which combines common techniques with fuzzy logic and neural networks. Such a mixed implementation leads to a more active control design with enhanced system performance and robustness. Whereas, classical control allows different design goals such as a steady-state and transient features of the closed-loop 
system to be specified. fuzzy logic and neural networks are integrated to overcome the problems with uncertainties in the system parameters and topology encountered in the classical model-based design. The advent of vector control techniques has slightly solved induction motor control problems; because they are oversensitive to variations drive parameter and performance may depreciate if conventional controllers are used. Fuzzy logic and neural network based controllers are considered as a potential candidate for such an application [23].

Installed a Variable Speed Drive system to control to starting of motor in both cases either standalone or with a full mechanical load. Here is illustrated a rough estimation with compared to the investment cost for direct online connected and VFD in application with a small pump.

Table 1. Comparisons between costs saving using small VF-Drive in pump application [3]

\begin{tabular}{l|l|l}
\hline \multicolumn{2}{l}{ Direct online (DOL) connected pump } & \multicolumn{2}{l}{ Efficient solution with VFD } \\
\cline { 1 - 2 } Pump and Motor $(\sim 3 \mathrm{~kW})$ & 1000 Euro & 1000 Euro \\
Installation & 1000 Euro & Installation; 1200 Euro + VFD 800 Euro \\
Total cost DOL & 2000 Euro & Total with VFD: 3000 Euro \\
\cline { 1 - 1 } Energy 15 years & (assumed 30\% energy savings) \\
\cline { 1 - 2 } Consumption with DOL & $394200 \mathrm{kWh}$ & $275940 \mathrm{kWh}$ \\
Energy cost with DOL $(9$ sent $/ \mathrm{kWh})$ & 35478 Euro & 24834 Euro \\
\hline
\end{tabular}

\subsection{Centrifugal Load}

Variable Speed Drives offer a better solution for energy saved to centrifugal loads such as pumps, compressors and fans. Therefore, centrifugal load equipment subjected to set of speed laws. An affinity law defines the relationship between compressor pressure to pump head, flow rate to rotor shaft speed or rotor speed system mechanical power consequently, all These variable can be expressed through the affinity law [24], the Figure 4 shows the parameters by apply affinity law for Pump, fan in both axial and centrifugal flows [3].
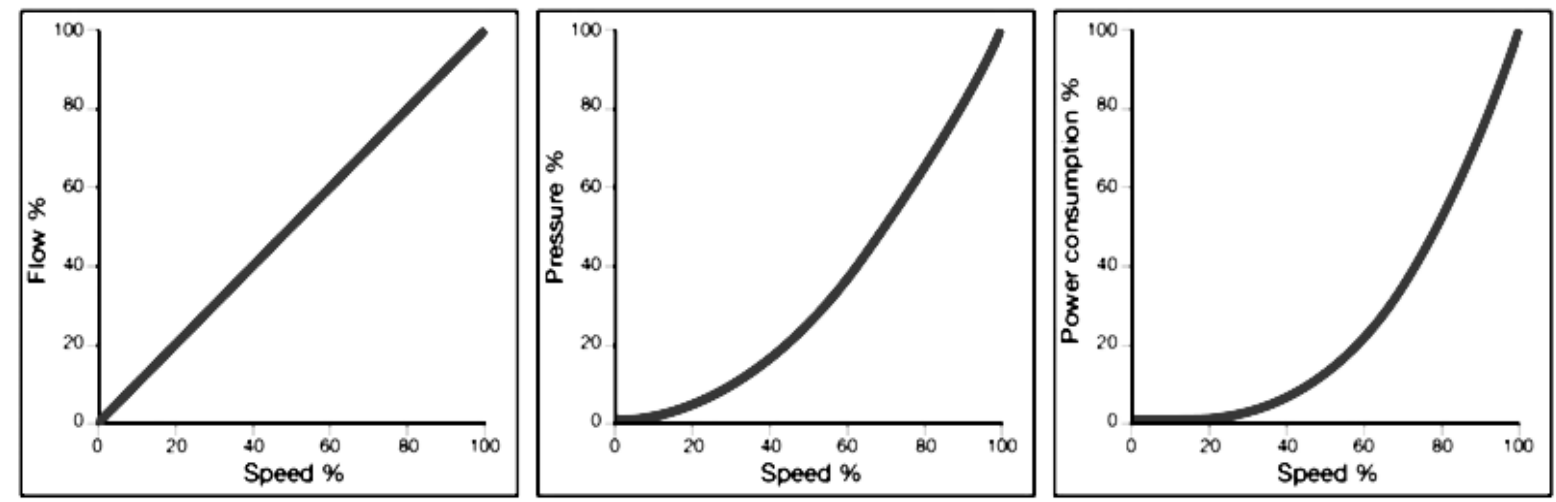

Figure 4. Block Affinity laws express relationship between Variables.

According to affinity laws, the relationship between the changing in pressure with flow rate or speed leads to power change in correlation to flow rate. Whereas flow rate changed linearly with speed although the compressors pressure is proportional to the square of shaft speed or flow rate. At the same time the power required are commensurate to the cube of the rotor speed or flow rate. The latter case is most important, because, when the rotor speed regress the electrical power consumption reduced by the cubic. Hence, the less reduced in rotor speed can provided saving in consume power. This can be seen in example Figure 4 . At $75 \%$ of shaft speed; this provides $75 \%$ of the flow rate, in addition it use only 
$42 \%$ of the power needed to generate a full flow rate. When the flow rate is regressed to $50 \%$ with respect to speed, as well as the power consumption also reduced to $12.5 \%$.

$$
\begin{aligned}
& \frac{\text { flow }_{1}}{\text { flow }_{2}}=\frac{N_{r 1}}{N_{r 2}} \\
& \frac{H_{1}}{H_{2}}=\left(\frac{N_{r 1}}{N_{r 2}}\right)^{2} \\
& \frac{P_{1}}{P_{2}}=\left(\frac{N_{r 1}}{N_{r 2}}\right)^{3}
\end{aligned}
$$

Where: $\mathrm{N}_{\mathrm{r} 1}$ : Shaft speed, $\mathrm{N}_{\mathrm{r} 1}$ required speed (RPM) $\mathrm{P}_{\mathrm{r} 1}$ : the machine hours power $\mathrm{P}_{\mathrm{r} 2}$ : required hours power. $\mathrm{H}_{1}, \mathrm{H}_{2}$ Hydraulic Head in meter it particular measurement of liquid pressure, over a geodetic datum when using a VFD to Driving a centrifugal pump, from the relation in the second equation of Affinity Law we can determine the pump speed required to develop h1.

\section{EXPERIMENTAL STUDY}

Employ Inverter to control speed of Ac motors is not an innovation, but in this study introduces a new technique for implementing of AC Drive. It has made them even more attractive due to their good price and easier to assemble. Therefore, it is very important to examine behavior and performance of this model. The experimental setup built in laboratory conditions, three-phase induction motor used as load, which are gives real operation characteristics and results. As mentioned before, the study includes the performance from point of view input power $(\mathrm{W})$, energy consumption $(\mathrm{J})$.

Implementation of traditional AC-Drives and PWM inverter is simple, because it depends on some ordinary analogue electronic circuits, like triangle carrier wave generator, operational amplifiers. Which are simple components. Whereas, new technique for designing of Variable Frequency Drive, it has made them even more efficient. The proposed design is totally based on interconnection module technique, which is available in the markets nowadays. The real time model for AC Drive system appearance in the block diagram in Figure. 5 and the fully assembled to the AC Drive system is shown in Figure 7.

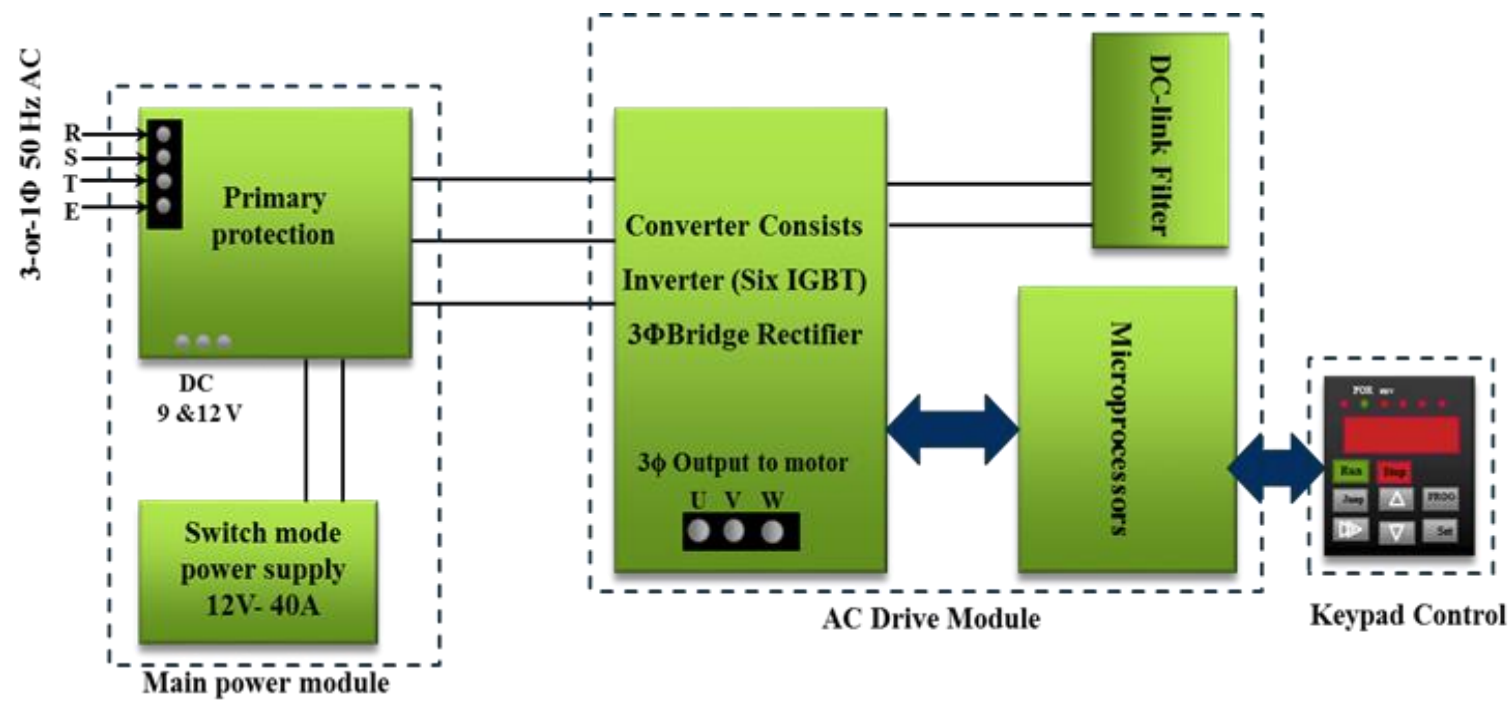

Figure 5. Block diagram of AC drive model. 
Because of the rapidly switching frequency into a power inverter circuit produces an increase in temperature for the six semiconductor devices, which is lead to weaker the performance of semiconductor switches, as well as unsteady running to overall system components. In order to keep the system temperature at normal limits and avoids overheating in the overall system. The 'switch mode power supply module' has been use to supply cooling system. The module obtains pure and stable DC $(+9,+12$ Volt $)$ and not affected by interference. A cooling power supply module based on the inverter shown in Figure 7.

To improve the diagnostic system technique, under fault conditions, protection module is used in order to conserve the system under fault "short circuit" and to make ensures to protect components of an electrical circuit. The protection module based into four Cartridge Fuses. Three (10A) are used to protect the inverter circuit and $(2 \mathrm{~A})$ is used to protect a $(+9 \&+12 \mathrm{Volt}$ DC) power supply. The practical protection circuit shown in Figure 8.

\subsection{Measurement Setup}

In order to measure the energy between supply and load side. The experimental tests consist of two parts. The first part is by direct attached of three-phase induction motor to the sinusoidal supply, without installing VFD model. Results obtained by running the electric motor, at different periods. The second part is by installing a practical model of AC Drive. The laboratory test scheme for direct on-line connecting is shown in Figure7. The Figure 8 illustrates the laboratory test scheme when installing a practical design of AC Drive. Two tests applied to the motor under no- load condition. The specification of motor listed in Table 2.

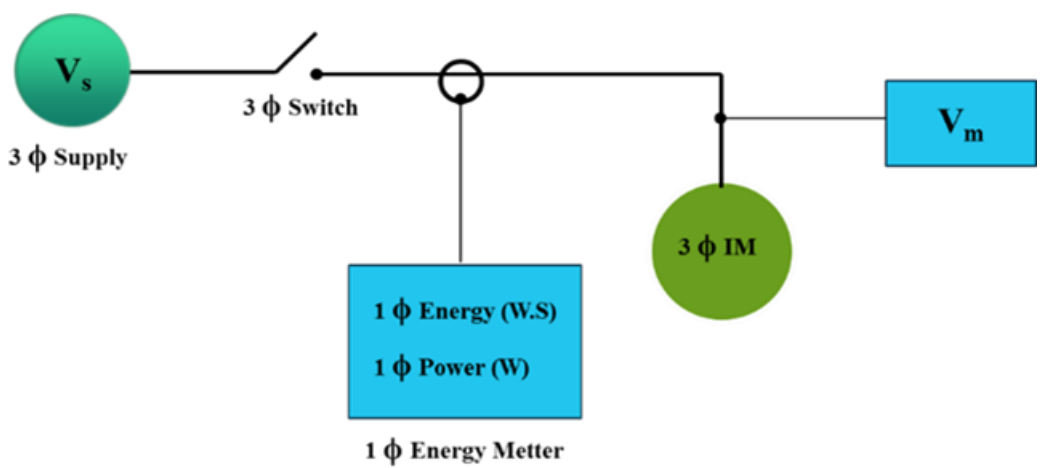

Figure 6. Block diagram of under laboratory test for direct on-line connecting.

Where; $\mathrm{V}_{\mathrm{s}}$ : Three phase laboratory source voltage at rated frequency $50 \mathrm{~Hz} \mathrm{~V}_{\mathrm{m}}$ : Voltage at terminals "line Voltage" of motor, obtained by using voltmeter And the other parameters (Energy, Power) are obtain by using Energy-Metter device.

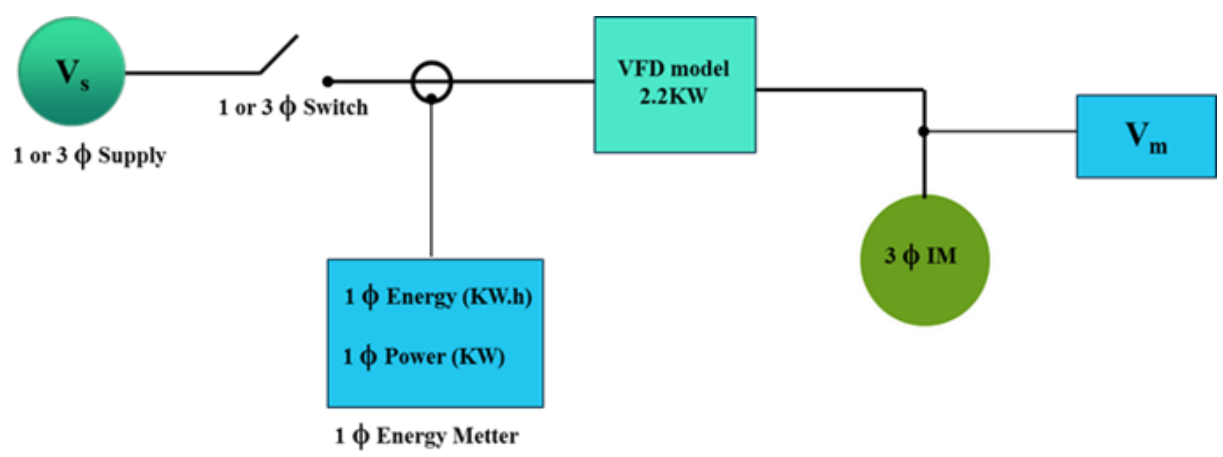

Figure 7. Block diagram of when installing AC drive model under laboratory test. 
Table 2. The specification of three-phase induction motor.

\begin{tabular}{l|l}
\hline Number of phase & 3 \\
\hline Motor Power & $1 \mathrm{Hp}$ \\
Frequency & $50 \mathrm{~Hz}$ \\
Number of Poles & 4 \\
Volts $(\Delta)$ & $230 \mathrm{~V}$ \\
Rated current $(\Delta)$ & $3.6 \mathrm{~A}$ \\
Motor Speed & 1420 R.P.M \\
Efficiency & $79 \%$ \\
PF & 0.67 \\
\hline
\end{tabular}

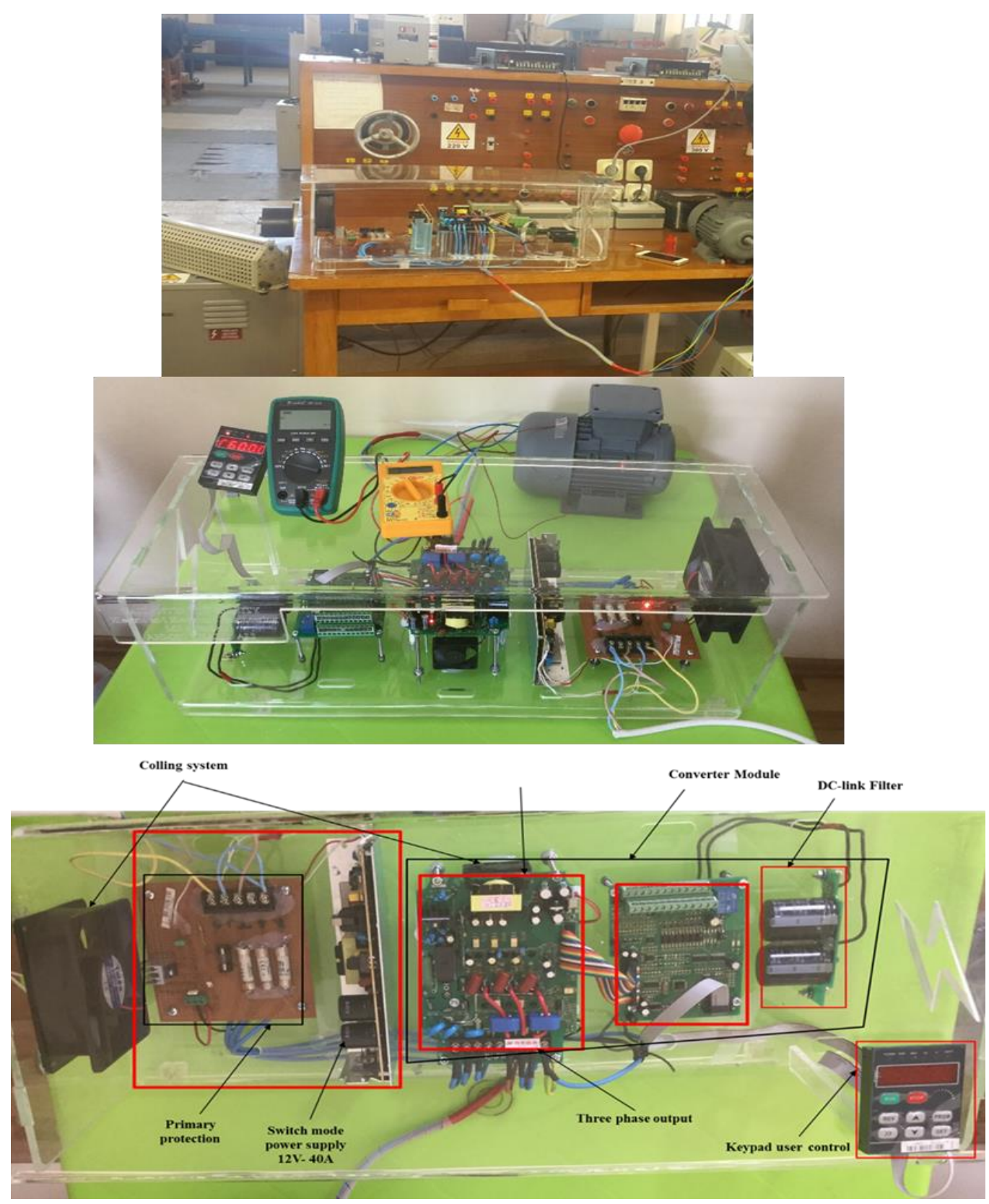

Figure 8. Picture to describe the parts of AC Drive model as well as the final assembly for the AC-Drive model, also snapshot to laboratory test. 


\subsection{Results recorded from two tests}

Applying three phase AC $380 \mathrm{~V}$ (star connection), $50 \mathrm{~Hz}$ to the motor the results in Table 3 obtained the direct- online operation of three- phase asynchronous motor.

Table 3. Results of direct online operation of 3-phase Induction motor

\begin{tabular}{l|l|l}
\hline Time(hour) & Energy(KJ) & $\mathrm{P}(\mathrm{KW})$ \\
\hline 1 & 946.8 & \\
2 & 1893.6 & 0.26 \\
3 & 2840.4 & \\
\cline { 2 - 3 } $\mathrm{V}_{\mathrm{m}}(\mathrm{V})$ & 380 & \\
\hline
\end{tabular}

The results are obtained after a few seconds of running motor.

By applying single phase AC $220 \mathrm{~V}, 50 \mathrm{HZ}$ to the input of AC- Drive model. And set the inverter carrier frequency $(8 \mathrm{KHz}$ ) and operation frequency $50 \mathrm{~Hz}$ (which is applied to the motor), after a few seconds of running. The data can be recorded through Table 4 and Figure 10. The Figure. 9 demonstrate clearly the energy saved practically when install the implementation AC-Drive model.

Table 4. The results when installing AC drive model.

\begin{tabular}{l|l|l}
\hline Time(hour) & \multicolumn{1}{l}{ Energy(KJ) } & $\mathrm{P}(\mathrm{KW})$ \\
\hline 1 & 546.4 & \\
2 & 1092.9 & 0.151 \\
3 & 1639.3 & \\
\cline { 2 - 3 } $\mathrm{V}_{\mathrm{m}}(\mathrm{V})$ & 230 & \\
\hline
\end{tabular}

In order to estimate the investment cost during one year, if installing the proposed model, we will postulate that same practical motor used in above tests, running 3 hours per day. In addition, electricity cost (0.39TL kilowatts) From above results it can calculate the energy cost in Turkish Liras (T.L) per year [25].

$$
\text { Enargy Cost }=K W * \text { hour } * T L / K W
$$

Table 5 Calculations of energy cost per year.

\begin{tabular}{ll}
\hline Test state (3- hours per day) & Energy Cost per year in (T.L ) \\
\hline Direct online connected (1-Hp motor) & 130 \\
Using designed model (1-Hp motor) & 73 \\
\hline
\end{tabular}




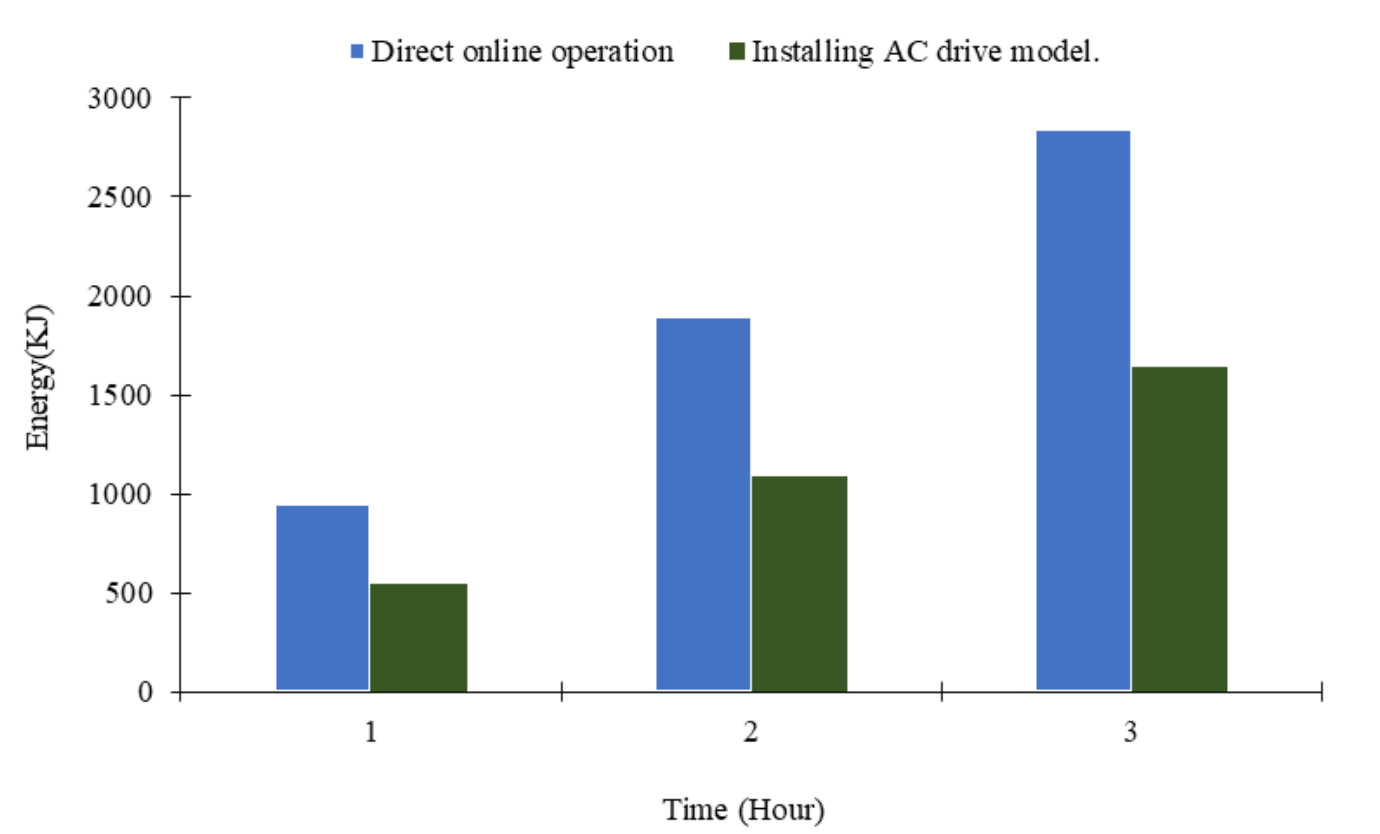

Figure 9. Comparison between energy drawn from grid to the three-phase induction motor in both cases; Direct-online connection and when install the model of AC-Drive.

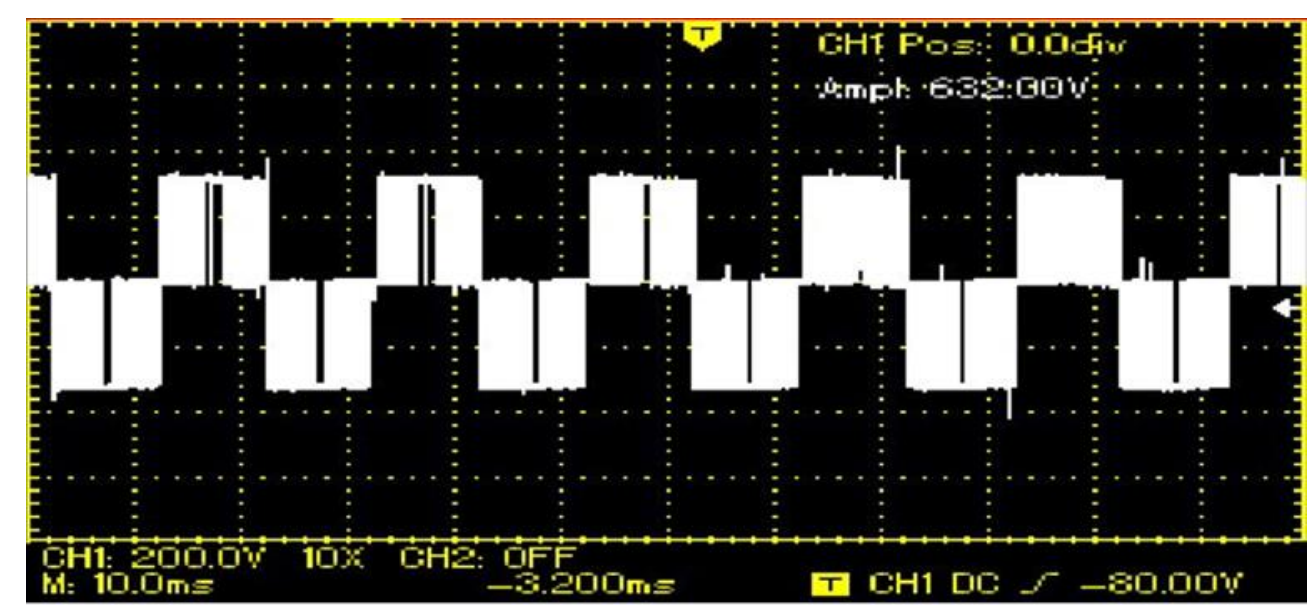

Figure 10. Waveform of "line Voltage" at terminals of "IM" when install implementation model under laboratory test.

To understand exactly the concept of energy saving when installing the inverter fed to squirrel cage three-phase induction motor. It's very useful to make comparisons between two tests. From above results, the following notices can be recorded.

1) It can be seen clearly a power consumption when installing the practical model of VFD it's excellently reduced, with respect to the direct on-line connect.

2) Through the results the energy consumption also reduces. When running a long time, consequently, more help to energy savings and reductions in $\mathrm{CO} 2$ emission. [26]

3) The operating cost per year of the electric motor much reduced when installing the AC Drive, which is that a more economic to the customer. 


\section{CONCLUSIONS}

Employ inverter to speed control of single or three-phase squirrel cage induction motor, is not an innovation. Whereas the new strategy to implementation of this device it has made even more practically, due to their moderate price, easy to construct. This study focused to help understand the concept of energy savings when installing a proposed design of the AC-Drive system fed a 3-phase cage motor. The results due to experimental indicate that AC Drive model is accurate and practicable and there is much energy reserved when used a proposed design for both small and large-scale electrical motors.

From the above conclusion and due to experimental results the implementation model AC Drive it can be recommended to real time applications due to their uncomplicatedness, robustness and simplified for regulation. However, the model can be considered as the best and the optimum for speed control of squirrel cage motor and help to reduce energy use, even more, it's one of the efficient solutions for energy saving and reduce $\mathrm{CO} 2$ emissions to the air.

\section{REFERENCES}

[1] Muhammad, H.R.. Power Electronics-Circuits, Devices, and Applications. Upper Saddle River, NJ, Pearson Prentice Hall, 2004.

[2] Bose, B.K. Power electronics and motor drives: advances and trends. Elsevier, 2010.

[3] Ristimaki, T. Energy efficiency through variable frequency drives. Honeywell. August, 2008.

[4] Tang, Y., Xu, L. A flexible active and reactive power control strategy for a variable speed constant frequency generating system. IEEE Transactions on power electronics, 1995, 10.4: 472-478 < https://doi.org/10.1109/63.391945>

[5] Járdán, R.K, et al. Laboratory system for measurement of iron losses in high speed drives. International Journal of Hydrogen Energy, 2016, 41.29: 12650-12658< https://doi.org/10.1016/j.ijhydene.2016.01.061>

[6] Teitel, M., et al. Energy saving in agricultural buildings through fan motor control by variable frequency drives. Energy and Buildings, 2008, 40.6: 953-960 < https://doi.org/10.1016/j.enbuild.2007.07.010>

[7] Saidur, R., et al. Applications of variable speed drive (VSD) in electrical motors energy savings. Renewable and Sustainable Energy Reviews, 2012, 16.1: 543-550.< https://doi.org/10.1016/j.rser.2011.08.020>

[8] De Almeida, A.T.; Ferreira, F.J.T.E., Both, D.. Technical and economical considerations in the application of variable-speed drives with electric motor systems. IEEE Transactions on Industry Applications, 2005, 41.1: 188-199 < https://doi.org/10.1109/TIA.2004.841022>

[9] Irvine, G., Gibson, I.H. The use of variable frequency drives as a final control element in the petroleum industry. In: Industry Applications Conference, 2000. Conference Record of the 2000 IEEE. IEEE, 2000. p. $2749-2758<$ https://doi.org/10.1109/IAS.2000.883212>

[10] Agamloh, E.B. Power and Efficiency Measurement of Motor-Variable-Frequency Drive Systems. IEEE Transactions on Industry Applications, 2017, 53.1: 766-773< https://doi.org/10.1109/TIA.2016.2602807 >

[11] Panasetsky, D., et al. Simplified variable frequency induction-motor drive model for power system stability

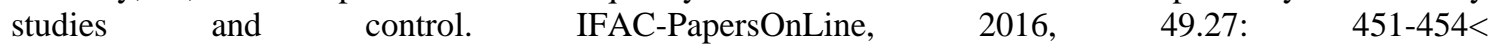
https://doi.org/10.1016/j.ifacol.2016.10.774>

[12] Shankar, V.K.A., et al. A comprehensive review on energy efficiency enhancement initiatives in centrifugal pumping system. Applied Energy, 2016, 181: 495-513< https://doi.org/10.1016/j.apenergy.2016.08.070>

[13] Wang, G.; Kiamehr, K.; Song, L. Development of a virtual pump water flow meter with a flow rate function of motor power and pump head. Energy and Buildings, 2016, 117: 63-70< https://doi.org/10.1016/j.enbuild.2016.02.003>

[14] Sueker, K.H. Power electronics design: a practitioner's guide. Elsevier, 2005.

[15] Holtz, J. Sensorless control of induction motor drives. Proceedings of the IEEE, 2002, 90.8: 1359-1394< https://doi.org/10.1109/JPROC.2002.800726>

[16] WEG Electrical Motors, "Induction motors fed by PWM frequency inverters," 2008. 
[17] Hurst, K.D., et al. A self-tuning closed-loop flux observer for sensorless torque control of standard induction machines. IEEE transactions on power electronics, 1997, 12.5: 807-815< https://doi.org/10.1109/63.622998>

[18] Trzynadlowski, A.M. Control of induction motors. Elsevier, 2000.

[19] Takahashi, I.; Ohmori, Y. High-performance direct torque control of an induction motor. IEEE Transactions on Industry Applications, 1989, 25.2: 257-264< https://doi.org/10.1109/28.25540>

[20] Rodriguez, J., et al. A novel direct torque control scheme for induction machines with space vector modulation. In: Power Electronics Specialists Conference, 2004. PESC 04. 2004 IEEE 35th Annual. IEEE, 2004. p. 1392-1397< https://doi.org/10.1109/PESC.2004.1355626>.

[21] Chung, P.Y.; Dölen, M.; Lorenz, R.D. Parameter identification for induction machines by continuous genetic algorithms. In: Proceedings ANNIE. 2000. p. 1-13

[22] Thomas, D.E.; Armstrong-Helouvry, B. Fuzzy logic control-a taxonomy of demonstrated benefits. Proceedings of the IEEE, 1995, 83.3: 407-421< https://doi.org/10.1109/5.364487>.

[23] Denai, M.A., et al. Fuzzy and neural control of an induction motor. Appl. Math. Computer Sci., 2002, 12.2: 221-234.

[24] Prachyl, S. Variable frequency drives and energy savings. Texas A \& M University, White Paper, 2010.

[25] De Almeida, A.T.; Ferreira, F.J.T.E.; Both, D. Technical and economical considerations in the application of variable-speed drives with electric motor systems. IEEE Transactions on Industry Applications, 2005, 41.1: 188-199 <https://doi.org/10.1109/TIA.2004.841022>.

[26] Räsänen, J.-E.; Schreiber, E.W. Using Variable Frequency Drives (VFD) to save energy and reduce emissions in newbuilds and existing ships. White paper, ABB Marine and Cranes, 2012. 